\title{
(2) OPEN ACCESS \\ Overview of retrospective data harmonisation in the MINDMAP project: process and results
}

\author{
Tina W Wey (10, ${ }^{1}$ Dany Doiron, ${ }^{1}$ Rita Wissa, ${ }_{1}^{1}$ Guillaume Fabre, ${ }_{1}^{1}$ Irina Motoc, ${ }^{2}$

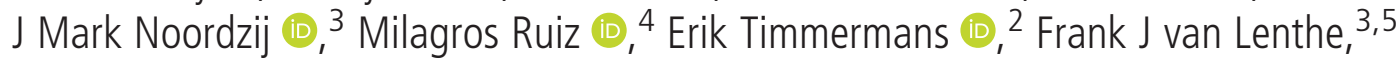 \\ Martin Bobak, ${ }^{4}$ Basile Chaix, ${ }^{6}$ Steinar Krokstad (10, 7,8 Parminder Raina, ${ }^{9,10,11}$ \\ Erik Reidar Sund $\mathbb{B}^{1},{ }^{7,8,12}$ Marielle A Beenackers, ${ }^{3}$ Isabel Fortier $^{1}$
}

- Supplemental material is published online only. To view please visit the journal online (http://dx.doi.org/10.1136/ jech-2020-214259).

For numbered affiliations see end of article.

\section{Correspondence to} Tina W Wey, Maelstrom Research, Research Institute of the McGill University Health Centre, 1650 Cedar Ave, Montreal, QC H3G 1A4, Canada;

twey@maelstrom-research.org

Received 3 April 2020 Revised 9 July 2020

Accepted 12 July 2020 Published Online First

12 November 2020

\begin{abstract}
Background The MINDMAP project implemented a multinational data infrastructure to investigate the direct and interactive effects of urban environments and individual determinants of mental well-being and cognitive function in ageing populations. Using a rigorous process involving multiple teams of experts, longitudinal data from six cohort studies were harmonised to serve MINDMAP objectives. This article documents the retrospective data harmonisation process achieved based on the Maelstrom Research approach and provides a descriptive analysis of the harmonised data generated. Methods A list of core variables (the DataSchema) to be generated across cohorts was first defined, and the potential for cohort-specific data sets to generate the DataSchema variables was assessed. Where relevant, algorithms were developed to process cohort-specific data into DataSchema format, and information to be provided to data users was documented. Procedures and harmonisation decisions were thoroughly documented. Results The MINDMAP DataSchema (v2.0, April 2020) comprised a total of 2841 variables (993 on individual determinants and outcomes, 1848 on environmental exposures) distributed across up to seven data collection events. The harmonised data set included 220621 participants from six cohorts (10 subpopulations). Harmonisation potential, participant distributions and missing values varied across data sets and variable domains.

Conclusion The MINDMAP project implemented a collaborative and transparent process to generate a rich integrated data set for research in ageing, mental wellbeing and the urban environment. The harmonised data set supports a range of research activities and will continue to be updated to serve ongoing and future MINDMAP research needs.
\end{abstract}

\section{INTRODUCTION}

The MINDMAP (promoting mental well-being and healthy ageing in cities) project offers a multinational data infrastructure to help investigate the opportunities offered by urban environments for the promotion of mental well-being and cognitive function of older individuals. This infrastructure allows multiple investigators to securely and remotely analyse harmonised cohort study data across European and Canadian populations. It also provides approved MINDMAP researchers access to the longitudinal data items and statistical power required to investigate direct and interactive effects of social, environmental and lifestyle determinants of mental health outcomes across different cities, to facilitate both comparative work and pooled analysis of outcomes. To create the MINDMAP infrastructure, cohort-specific data had to be harmonised (processed under a common format allowing co-analysis of data across studies), which is important for ensuring content equivalence and reducing bias due to methodological differences, but presents multiple challenges. ${ }^{1}$ Population sampling frames, participant followups, types of information collected, and variable formats and content vary extensively across cohort studies. It was thus essential to implement a rigorous process to harmonise, integrate and document the core data to be generated.

MINDMAP implemented such a process, ${ }^{2}$ and its current data platform supports a broad range of research activities. The harmonisation teams comprised multiple research groups with a wide range of expertise, who contributed to harmonising data from six population-based cohort studies ${ }^{3-8}$ across Western and Eastern European countries and Canada. The current article details the harmonisation process and provides a descriptive analysis of an early version of the MINDMAP data set (v2.0, April 2020). It describes the harmonisation procedures, summarises variables that were harmonised across studies and provides an overview of key information useful for understanding results presented in this special issue and by future projects using the MINDMAP data set. More broadly, this article illustrates challenges in retrospective harmonisation and considerations for promoting a transparent process to produce collaborative data resources.

\section{METHODS}

The harmonisation process was informed by the retrospective harmonisation guidelines ${ }^{1}$ and used the open-source software Opal 2.16 and Mica 3.9 developed by Maelstrom Research. ${ }^{9}$ The harmonisation teams were composed of epidemiologists, social scientists and statisticians from specialised scientific domains. University College London (UK) harmonised mental health outcomes; VU University Medical Center Amsterdam (Netherlands) harmonised social factors and perceived environment variables, and linked and derived social-environmental variables; the Research Institute of the McGill University Health 
Centre (Canada) with Erasmus University Medical Center (Netherlands) harmonised sociodemographic, lifestyle and behavioural, and health variables; and Erasmus University Medical Center linked and derived physical-environmental variables. All harmonisation work was done in collaboration across as well as within subject areas to ensure a consistent and coordinated process, and the harmonisation team had weekly to monthly videoconference calls as needed throughout the process.

\section{Individual-level determinants and outcomes data}

The process to harmonise data on individual risk factors (eg, sociodemographic characteristics, life habits) and physical and mental health status across participating cohorts included the following steps.

1. Assemble cohort-specific information and select studies

For each MINDMAP participating cohort, study designs (eg, number of data collection events, population sampling frame, participant selection criteria) and variable data dictionaries were gathered and catalogued online following Maelstrom Research standards. ${ }^{10}$ English versions of documentation were available for all studies, and any ambiguities in language were clarified with the cohort study teams. Six studies (including 10 subpopulations) were included in this version of the MINDMAP data set (v2.0, April 2020) (table 1). Three studies that are part of

\begin{tabular}{lllll}
\hline Table 1 & Overview of MINDMAP participating cohort designs and subpopulations included in the harmonisation \\
\hline Subpopulation & $\begin{array}{l}\text { Participants } \\
(\mathbf{n})^{*}\end{array}$ & Country & Recruitment & Data collection mode \\
\hline CLSA_COP & 30097 & Canada & $\begin{array}{l}\text { Provincial health registries and telephone } \\
\text { sampling using random digit dialing of } \\
\text { residents within 25-50 km of 1 of 11 data } \\
\text { collection sites across seven Canadian } \\
\text { provinces (Alberta, British Columbia, } \\
\text { Manitoba, Nova Scotia, Newfoundland and } \\
\text { Labrador, Ontario, Quebec) }\end{array}$ & $\begin{array}{l}\text { In-depth interview in participants' } \\
\text { homes; physical and biological } \\
\text { measurements at data collection sites }\end{array}$ \\
CLSA_TRA & 21241 & Canada & $\begin{array}{l}\text { Canadian Community Health Survey (CCHS) - Telephone interview } \\
\text { Healthy Aging cycle 4.2, provincial health } \\
\text { registries and telephone sampling using } \\
\text { random digit dialing across the 10 Canadian } \\
\text { provinces }\end{array}$
\end{tabular}

\begin{tabular}{lcll} 
GLOBE & 22721 & Netherlands & $\begin{array}{l}\text { Municipal registries of the city of Eindhoven } \\
\text { and } 15 \text { surrounding villages in the Southern } \\
\text { part of the Netherlands }\end{array}$ \\
\hline HAPIEE_CZ & 8857 & $\begin{array}{l}\text { Czech } \\
\text { Republic }\end{array}$ & $\begin{array}{l}\text { Population registers from Havirov/Karvina, } \\
\text { Hradec Kralove, Jihlava, Kromeriz, Liberec and } \\
\text { Usti nad Labem }\end{array}$
\end{tabular}

$\begin{array}{lccc}\text { HAPIEE_LT } & 9360 & \text { Lithuania } & \text { Population registers from Kaunas } \\ \text { HAPIEE_RU } & 7151 & \text { Russia } & \text { Population registers from Novosibirsk }\end{array}$

$\begin{array}{lll}\text { HUNT } & 106429 \quad \text { Norway } & \begin{array}{l}\text { Postal invitation to all citizens of Nord- } \\ \text { Trøndelag County (24 municipalities) }\end{array} \\ \text { LASA1 } 3107 \quad \text { Netherlands } & \begin{array}{l}\text { Municipal registries from three geographic } \\ \text { regions: Amsterdam, Wormerland, Waterland } \\ \text { (three municipalities in the West), Zwolle, Ommen, } \\ \text { Genemuiden, Zwartsluis, Hasselt (North-East), and } \\ \text { Oss, Uden, Boekel (South); oversampling of older } \\ \text { people and older men in particular }\end{array}\end{array}$

RECORD $\quad 9821 \quad$ France
depth interviews for two subsamples regions: Amsterdam, Wormerland, Waterland (three municipalities in the West), Zwolle, Ommen, Genemuiden, Zwartsluis, Hasselt (North-East), and Oss, Uden, Boekel (South); oversampling of older people and older men in particular

Postal questionnaire (baseline); in(random and chronically ill)

Structured questionnaire at home: examination in clinic; face-to face computer-assisted personal interviewing (follow-up); death registers

Structured questionnaire in clinic examination in clinic; face-to face computer-assisted personal interviewing (follow-up); death registers

Structured questionnaire in clinic; examination in clinic; face-to face computer-assisted personal interviewing (follow-up); death registers

Questionnaires and physical and biological measurements taken at health examination sites in each municipality

Face-to-face interview; medical in-home $55-85$ years old interview; telephone interview

Face-to-face interview; medical in-home $55-65$ years old interview; telephone interview

Invitation to all clinic patients at general health check-ups from four Centre d'Investigations Préventives et Cliniques (IPC) centers (Paris, Argenteuil, Trappes, Mantes-la-Jolie) at baseline

45-69 years old

45-69 years old

45-69 years old

\section{$20+$ years old}

\section{Questic} physical and biological measurements during check-up

Inclusion/exclusion criteria

45-85 years old; able to give consent; excluding residents in the three territories, persons living on federal First Nations reserves and other First Nations settlements in the provinces, full-time members of the Canadian Armed Forces, and individuals living in institutions

45-85 years old; able to give consent; excluding residents in the three territories, persons living on federal First Nations reserves and other First Nations settlements in the provinces, full-time members of the Canadian Armed Forces, and individuals living in institutions

15-75 years old; non-institutionalised

30-79 years old; residing in 1 of the112 preselected municipalities; able to answer questions themselves or with minimal help in French

\footnotetext{
${ }^{*}$ This represents the total number of unique participants, which includes sample boosting in follow-ups for some cohorts (GLOBE, HUNT, LASA1, RECORD).

CLSA_COP, Canadian Longitudinal Study on Aging (CLSA) ${ }^{3}$ comprehensive (in-depth); CLSA_TRA, CLSA tracking (telephone interview); GLOBE, Health and Living Conditions of the Population of Eindhoven and Surroundings (Gezondheid en Levens Omstandigheden Bevolking Eindhoven en omstreken) ${ }^{4}$. HAPIEE CZ, The Health, Alcohol and Psychosocial Factors in Eastern Europe Study ${ }^{5}$ Czech Republic; HAPIEE_LT, HAPIEE_-Lithuania; HAPIEE_RU, HAPIEE-Russia; HUNT, Nord-Trøndelag Health Study (Helseundersøkelsen i Nord-Trøndelag) ${ }^{6}$ 1-2-3 Cohort; LASA1, Longitudinal Aging Study Amsterdam (LASA) ${ }^{7}$ first cohort; LASA2, LASA second cohort; RECORD, Residential Environment and CORonary heart Disease Study. ${ }^{8}$
} 
the MINDMAP consortium were not included in the harmonisation project due to inaccessibility of relevant study-specific individual participant data.

2. Define core variables and evaluate harmonisation potential

MINDMAP investigators collaboratively defined an initial targeted set of variables that they considered relevant for addressing selected research questions, through discussion and drawing on study teams' expertise and the Maelstrom catalogue to identify available data. The harmonisation teams then used study documentation and data dictionaries to examine cohort-specific information collected in greater detail, and, in collaboration with Maelstrom Research, generated the final list of core variables (ie, the harmonised variables to be generated across studies) and their specifications (the DataSchema), ${ }^{11}$ which was adjusted from the initial list of target variables based on the available study-specific data. The DataSchema includes, for each variable, the name, definition, format (eg, integer, decimal) and units (eg, years, drinks/ week), and rules for harmonisation were defined and documented. Rules for harmonisation refer to specifications for making decisions about whether and how particular variables are harmonised (eg, occurrence of angina must be diagnosed). Separate DataSchema variables were defined for baseline and each participant follow-up (data collection events). DataSchema variables targeting equivalent content at different time points were distinguished by the variable name suffix (eg, participant age was 'sdc_age_0' for baseline, 'sdc_age_1' for first follow-up, etc).

After finalising the DataSchema, the harmonisation teams assessed and documented the potential for each cohort-specific subpopulation to generate the DataSchema variables defined (ie, the harmonisation potential). ${ }^{11}$ Input from cohort teams (which included principal investigators, researchers and data managers, and could overlap with harmonisation team members) was regularly sought to address questions regarding missing metadata or unclear information. Harmonisation potential was considered 'complete' if cohort-specific variables were the same as the DataSchema or could be transformed to generate DataSchema variables. Harmonisation potential was deemed 'impossible' if relevant cohort-specific data were not collected or incompatible with DataSchema variable definitions. Variables were only retained in the DataSchema if they could be generated for two subpopulations or across two time points within a subpopulation. An example outlining harmonisation potential of cohort-specific variables and proposed algorithms to generate a DataSchema variable is provided in online supplemental table S1.

3. Process data under common format

Cohort-specific data required to generate the DataSchema variables were transferred to a central data server at Erasmus Medical Center, in accordance with consortium data-sharing policies. ${ }^{2}$ A central RStudio server allowed authenticated harmonisation team members to securely access and process cohortspecific data under the DataSchema format. ${ }^{12}$ The harmonisation teams assessed the quality of data provided for their domain of interest by checking univariate distributions and coherence among related variables (eg, skip patterns and consistency of participant responses among data collection events). Any questions were clarified with cohort study teams and documented. To explore representativeness of the populations, age and sex distributions at baseline were compared with national statistics from the same year for each subpopulation.

Harmonisation teams verified the harmonisation potentials attributed and, where relevant, developed algorithms (eg, online supplemental table $\mathrm{S} 1$ ) using $\mathrm{R}^{13}$ scripts to process cohortspecific data into the DataSchema format. Processing methods included direct mapping (target variable same as source variable), algorithmic transformation, calibration (converting units), rescaling and standardisation methods. ${ }^{1}$ In most domains, harmonisation was achieved predominantly through algorithmic transformations. More complex algorithms were required to account for longitudinal data in harmonising lifestyle and behavioural variables and health variables (eg, 'Ever smoked' or 'Ever had a stroke' in follow-ups used information from earlier data collection events). Mental well-being scores measured with different scales were harmonised by collapsing scores into quantiles or cases/non-cases based on cutoffs. Cognitive measures were harmonised using rescaling methods or by converting to z-scores. 4. Explore quality and content of harmonised data sets generated

Descriptive statistics were used to summarise final harmonisation statuses of DataSchema variables across cohort-specific data sets and scientific domains. First, to explore harmonised data content within each subpopulation, univariate distributions were generated for each DataSchema variable and reviewed for correct participant numbers, distributions and missing values, compared to the initial cohort-specific data provided. Harmonisation algorithms were validated for logic and script syntax, and correspondence of variable metadata with DataSchema specifications was verified. Outliers were retained but noted to inform researchers. Next, multivariate cross-checks were performed to validate coherence among related variables (eg, 'current average number of cigarettes smoked per day' only greater than 0 if 'currently smokes any tobacco product' is true). Finally, variability in participant distributions and missing values was examined across subpopulations. This helped identify additional corrections (eg, frequencies that were very different for one subpopulation revealed an error in cohort-specific coding) and variability across subpopulations to consider. Potential effects of factors such as cohort sampling and recruitment, data collection methods and harmonisation decisions on variable heterogeneity across subpopulations were explored (see online supplemental tables S4 and S5, figure S1) and will be part of ongoing discussions with researchers to improve utility of the harmonised data.

\section{Preserve and disseminate harmonisation products}

A MINDMAP work repository was created on Github to document harmonisation decisions and processing scripts, and an interface was created on the Maelstrom Research catalogue (https://www.maelstrom-research.org/mica/network/mindmap) to collate and disseminate information about the cohort-specific designs and variables collected, DataSchema variables generated and harmonisation potential across studies. Secure access to the harmonised data for approved MINDMAP researchers is managed through the Opal data repository.

\section{Area-level environmental-exposure data}

Information on social- and physical-environmental exposures came from publicly available resources and were linked to cohort participant data using residential locations of cohort participants. To protect participant privacy, a series of steps were taken to blind the geospatial information, and linked data including only participant ID and environmental-exposure data (without any geospatial information) were transferred to the MINDMAP central server. More details on the sources of information and procedures used to link data are available elsewhere. ${ }^{2} 1415$

Availability of area-level data was limited, and years of collection did not necessarily correspond to the timing of the cohorts-specific data collection events (figure 1). Arealevel data were thus linked using information collected at the closest year to each data collection event. Harmonisation of the environmental-exposure DataSchema variables followed 


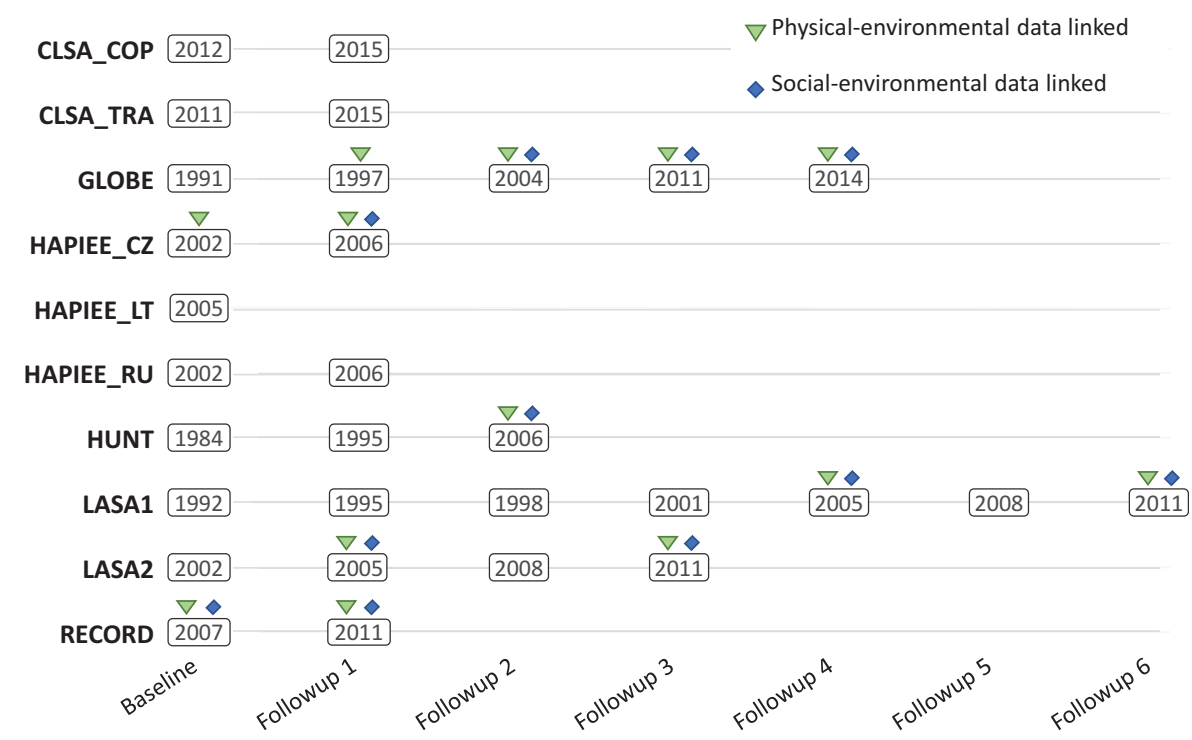

Figure 1 Overview of start years of data collection events in cohort studies and of time points with linked area-level information. Note that these do not reflect the time span of each data collection event.

the approach used for the individual-level cohort data. Processing of physical-environmental data and socialenvironmental variables to a common format included direct mapping and more complex transformations (eg, dichotomised variable of average income of area residents below/ above country-specific household mean).

\section{RESULTS}

\section{Cohort-specific data}

Cohort baseline data collection years ranged from 1984 to 2012, and the number of data collection events ranged from 1 to 7 (figure 1). The number of participants in each of the 10 cohort subpopulations ranged from 1837 to 106 429, with a total of 220621 participants (table 1). Participant sex distributions (overall $50.7 \%$ female, $49.3 \%$ male) were generally similar to contemporary age-matched national populations, with the exception of Residential Environment and CORonary heart Disease Study (RECORD), which recruited a larger proportion of males relative to the general French population $(34.5 \%$ female, $65.5 \%$ male at baseline) (table 2, figure 2). The participants median age at baseline was 56 years (range 14-102 years). Age distributions at baseline varied widely across sampled subpopulations, frequently differing from contemporary national populations (table 2).

\section{DataSchema variables and harmonisation potential}

The DataSchema (v2.0) included a total of 2841 variables: 993 from individual-level determinants and outcome data; 1848 from environmental-exposure data. As individual-level determinants and outcome variables were only defined for data collection events if they could be generated for more than one subpopulation or more than one data collection event within subpopulations, numbers differed across time points. There were 166 individual-level determinants and outcome variables at baseline and $165,159,139,134,112$ and 105 at follow-ups 1, 2, 3, 4, 5 and 6 , respectively. For environmental-exposure variables, 41 social-environmental and 223 physical-environmental (264 total) variables were defined for each time point. Online supple mental table S2 provides DataSchema information for baseline variables and administrative variables (data collection years and time intervals). Table 3 provides the distribution of DataSchema variables by domain and subdomain of information, following Maelstrom Research's cataloguing classification. ${ }^{10}$

The 10 subpopulations differed in the number of data collection events (from 1 to 7 ) and in the number of time points with linked area-level environmental-exposure data (from 0 to 4 ). This resulted in a total of 30 cohort-specific data collection events, 13 of these with linked area-level data on social- and/ or physical-environmental exposures (figure 1). Harmonisation potentials of DataSchema variables were evaluated only where applicable, that is, where the cohort collected data for that time point (eg, not considering sdc_age_6 for subpopulations without six follow-up events) and, for environmental-exposure variables, if area-level data were linked. This resulted in 8165 harmonisation potentials to evaluate. The overall percentage of complete harmonisation statuses was 63.0\% (5144 complete statuses/8165 evaluated). Individual determinants and outcome variables accounted for 4733 harmonisation statuses, of which 2523 (53.3\%) were complete and 2210 (46.7\%) were impossible. Environmental-exposure variables accounted for 3432 statuses, of which 2621 (76.4\%) were complete and 811 (23.6\%) were impossible. Harmonisation potential also varied across domains of information, ranging from $26.4 \%$ in life events, beliefs and values to $73.3 \%$ in physical measures and $78.6 \%$ in physical environment (table 3 ). Finally, harmonisation potential varied across subpopulations, ranging from $34.7 \%$ in HUNT to $80.6 \%$ in LASA1. All harmonisation statuses are presented in online supplemental table S3 and are also available on the Maelstrom catalogue. ${ }^{16}$ Complete statuses reflect harmonised variables achieved with processing methods ranging from direct mapping to complex algorithms using information and conditions from many cohort-specific variables, and the complexity of harmonisation algorithms required and any important decisions taken in harmonising each variable are available in RMarkdown files.

\section{Harmonised data content}

Participant distributions and missing values varied across the 10 subpopulations. For example, figure 2 presents participant distributions at baseline for several variables, including feeling 


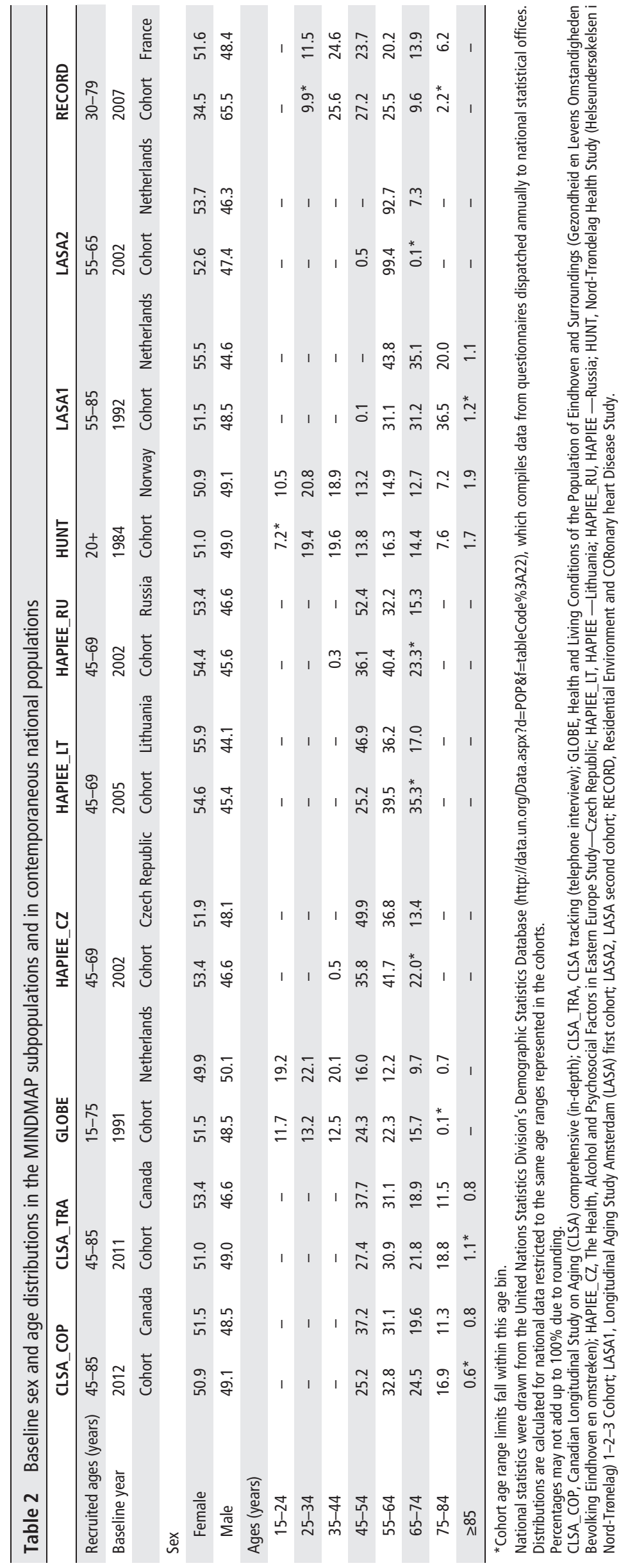


a) Age of the participant

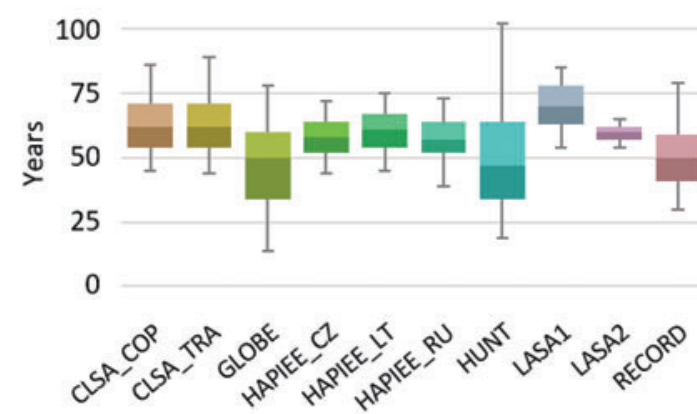

c)

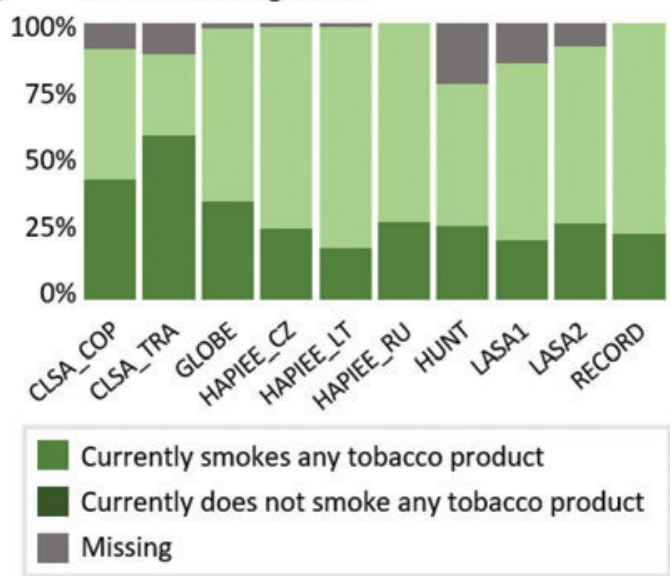

b)

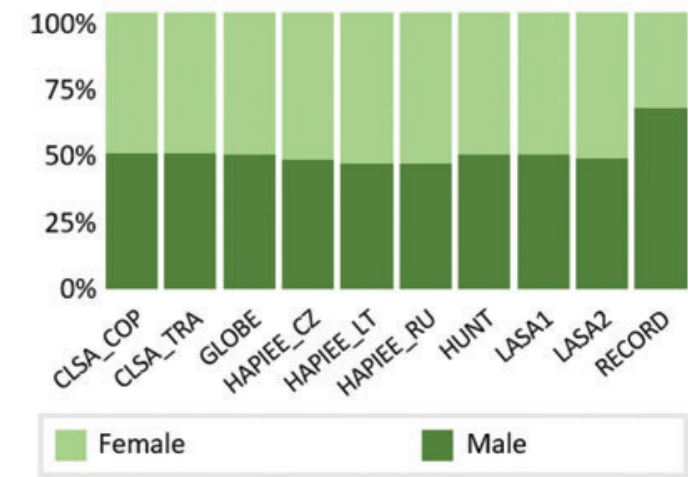

d)

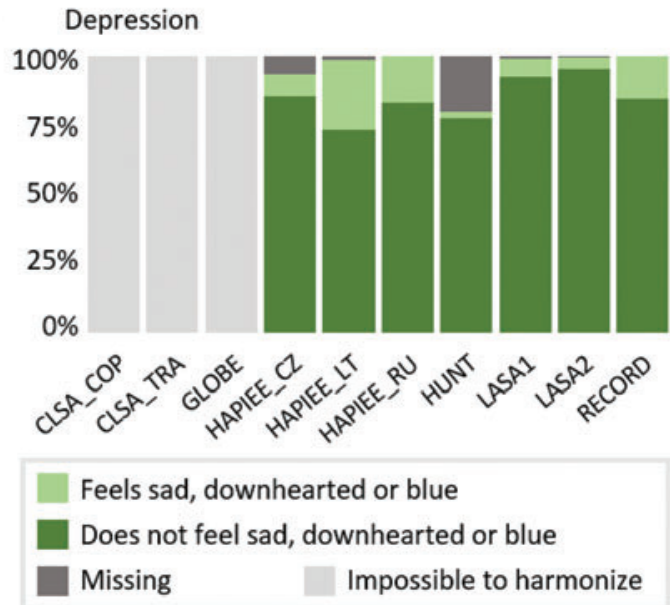

f) Highest level of education

e) Do you feel safe at night

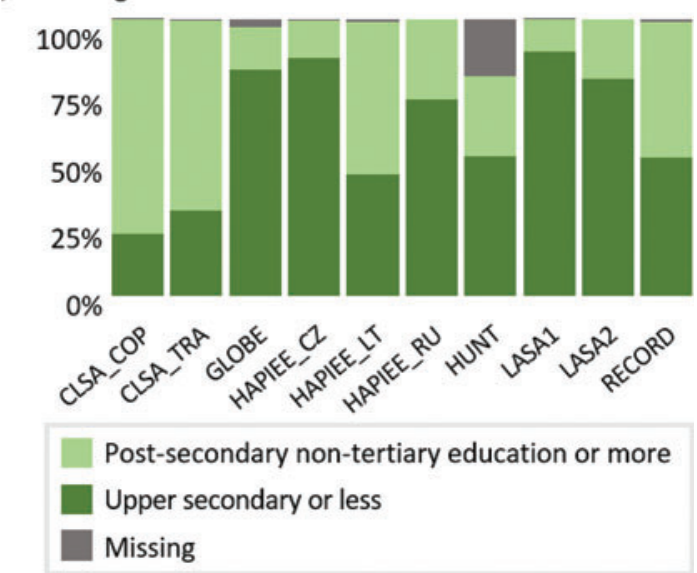

Figure 2 Participant distributions at baseline for selected harmonised variables. 'Impossible to harmonise' indicates variables that could not be harmonised for a subpopulation data set. 'Missing' indicates missing values within a subpopulation data set for variables with complete harmonisation status.

depressed, feeling safe at night, current smoking status and highest level of education. These illustrate variability in harmonisation potential, distribution of participants across categories, and percentage of missing data. Current smoking status and highest level of education could be created for all subpopulations, while depression was impossible for three subpopulations and feeling safe at night was impossible to generate for four. The percentage of participants with secondary-level education or more ranged from $11.4 \%$ in LASA 1 to $77.6 \%$ in CLSA_COP, and the percentage of missing data ranged from $0.2 \%$ in CLSA_COP to $42.5 \%$ in HUNT.
Note that missing values in harmonised data can result from missing values in cohort-specific data or harmonisation processing (eg, cohort-specific values of 'Don't know' and 'Prefer not to answer' were coded to missing in harmonised variables).

Various factors can explain the observed subpopulation variability including, but not limited to the population sampling frame, recruitment procedures, data collection methods and question format. An example of exploring the potential influence of such factors using a cognitive functioning variable is provided in online supplemental tables S4 and S5, figure S1. 
Table 3 Distribution of DataSchema variables and average per cent complete harmonisation potential by domains of information

Domain of information (N DataSchema variables per subdomain)

N DataSchema variables \% Complete

Sociodemographic and economic characteristics

(\% total) statuses

Age/birthdate (7), sex/gender (7), marital status (14), family and household structure (20), education (10), residence (28), labor $120(4.2)$ 64.1 force and retirement (17), income, possessions and benefits (17)

Lifestyle and behaviours

Tobacco (45), alcohol (37), nutrition (16), physical activity (73), sleep (12) and leisure activities (7)

Perception of health, quality of life, development and functional limitations

$55(1.9)$

71.4

Perception of health (21), quality of life (24) and functional limitations (10)

Diseases, ICD-10

Circulatory system disease (19), endocrine, nutritional and metabolic diseases (12)

Medication and supplements

Medication and supplement intake (15)

Physical measures and assessments

Anthropometry (91)

Life events, life plans, beliefs and values

Life events (33)

Cognition, personality and psychological measures and assessments

$171(6.0)$

52.7

Cognitive functioning (84), psychological distress and emotions (81), other psychological measures and assessments (6)

Social environment and relationships

Social network (42), social participation (110), social support (50) and other social environment characteristics (321)

Physical environment

Housing characteristics (7), built environment/neighborhood characteristics (1592)

Administrative information

$13(0.5)$

100.0

Date and time (13)

Total

$2841(100)$

ICD-10, International Statistical Classification of Diseases and Related Health Problems, 10th Revision.

\section{Documentation of harmonised data sets}

Information about the MINDMAP project, participating cohort designs and harmonisation potential is available on the Maelstrom Research catalogue. ${ }^{16}$ The web interface includes the capacity to search cohort-specific and DataSchema variables and documents data harmonisation potential across cohort subpopulations. In addition, harmonisation outputs and annotated $\mathrm{R}$ processing scripts are available on the MINDMAP GitHub repository. ${ }^{17}$ As documentation is updated regularly to reference the most recent version of harmonised data sets, current online information will vary from the information reported in this article.

\section{DISCUSSION}

MINDMAP implemented a rigorous multinational collaborative process to generate a large harmonised data set, which serves as a valuable resource for research on urban environments and mental health in ageing adults. The current harmonised data set (v2.0) includes 2841 harmonised variables from 30 data collection events across six cohort studies (including 10 subpopulations). The breadth of information, diversity of cohort studies and availability of longitudinal and environmental data are important strengths of the project. The success of the harmonisation process depended on the collaborative work of several international research teams contributing methodological and content expertise (cognition, environmental exposure, etc). This collaboration was enacted through a rigorous methodological approach and close communication among the domain experts, Maelstrom Research team and cohort study teams. In parallel, the technological infrastructure implemented allowed the international teams to work remotely on a central server to harmonise data, while protecting participant privacy, and now provides investigators with an efficient means to readily access and analyse the harmonised data set. ${ }^{2}$

The MINDMAP-harmonised data supports exploration of the impact of social and physical environments from 10 subpopulations in seven countries, which is quite novel and critical for studying the influence of urban environment on healthy ageing. Including diverse urban populations provides a wide range of exposures to inform how structural differences between countries or cities influence mental wellbeing and health. While not all core variables could be created across all cohort-specific data sets (the global harmonisation potential was $62.8 \%$ ), the data set generated supports valuable subanalysis across selected variables and/or studies (eg, analyses to date by JM Noordzij et al 2020, M Ruiz et al 2020, EJ Timmermans et al 2020). However, the utility for each research question needs to be carefully examined, and it is essential to recognise the potential and limitations of the data used. Researchers using the harmonised data set should consider multiple potential sources of subpopulation variability, where relevant, for their specific research needs. For example, where data collection methods and harmonisation processing vary among cohorts, heterogeneity in variable distributions could reflect a combination of underlying subpopulation differences and methodology, which could affect decisions such as selecting data to analyse, choosing an analytical approach and interpreting subpopulation heterogeneity.

The harmonised data generated presents important limitations. Definition of the target variables required a balance between ensuring integrity of scientific content (being as 
homogenous as possible across studies) and the need to allow a certain level of heterogeneity (combining information collected in different formats). ${ }^{18}$ These trade-offs were apparent, for example, in mental well-being and cognitive performance variables. Choosing to harmonise only information from identical scales provides more homogeneity but, as cohorts rarely use common scales, results in including fewer studies. On the other hand, using methods that increase the potential to integrate information across cohorts (eg, applying cut-offs, standardisation models) generally results in loss of information, increased heterogeneity and reduced ability to examine certain population differences. ${ }^{19} 20$ Creation of the initial DataSchema (v2.0) attempted to find this balance, but further exploration of the data content remains essential to better understand the quality of the variables generated. ${ }^{21-24}$ This will entail updates of the harmonised data set, optimising data content to better support current and upcoming research needs.

Several additional factors should be considered by investigators aiming to understand and use MINDMAP data. These factors include, but are not limited to, the following. First, subpopulation backgrounds, sampling frames, recruitment procedures and data collection profiles are, as expected, different. Second, subpopulations differ in their representativeness of national populations. Summaries in the current article come from raw sample data, but researchers should consider adjustments for analysis and inference about underlying populations. For example, CLSA data were designed to be analysed as one cohort (rather than the two samples presented here for harmonisation purposes) and with sampling weights (inflation weights for descriptive analysis and analytic weights for statistical testing). Third, the quality and variable resolution of the cohort-specific data provided varied. For example, outliers were noted but left in the data set, and the impact of missing values was not examined. Fourth, the number and timing of data collection events varied across cohorts. Longitudinal data offers important advantages over cross-sectional analyses but introduces other complexities to be considered during analyses. ${ }^{25}$ Interpretation of results must be made in consideration of such factors.

The MINDMAP process followed Maelstrom Research guidelines for rigorous retrospective data harmonisation, ${ }^{19-11}$ which have also been used by other retrospective harmonisation endeavours across population-based studies. ${ }^{26-29}$ The approach ensured generation of comprehensive and searchable documentation, including (1) cohort-specific designs and variables collected; (2) definition and characteristics of the DataSchema variables; (3) harmonisation potential across studies; and (4) algorithms used to process cohort-specific data into DataSchema variables. We hope that the information provided will help to properly understand, optimally use and further develop the MINDMAP data set.

\section{CONCLUSION}

The MINDMAP team implemented a collaborative and transparent process to generate a valuable harmonised data set to be used for research on ageing and mental well-being across different country and urban contexts. The current article describes the harmonisation process and harmonised data generated. More broadly, it provides an example of how large multinational collaborations can successfully implement and document retrospective harmonisation to generate valuable epidemiological data sets.

\section{What is already known on this subject}

Retrospective data harmonisation is important for achieving or improving comparability of similar data items collected by different studies. It enables leveraging existing cohort data resources to address research questions that are difficult or impossible to address in single studies and has become an important tool in collaborative research initiatives.

- Harmonisation of existing data raises major challenges, and transparent and thorough documentation of the harmonisation process is required for researchers to understand and use harmonised data.

\section{What this study adds}

- The MINDMAP team harmonised data from six international cohort studies to examine the individual and environmental determinants of mental well-being in older adults in diverse urban populations.

- This article describes the collaborative harmonisation process implemented and serves to inform researchers on how large multinational collaborations can successfully implement and document retrospective harmonisation to generate valuable epidemiological data sets.

\section{Author affiliations}

${ }^{1}$ Maelstrom Research, Research Institute of the McGill University Health Centre, Montreal, Canada

${ }^{2}$ Department of Epidemiology and Biostatistics, Amsterdam UMC, VU University Medical Center, Amsterdam Public Health Research Institute, Amsterdam,

Netherlands

${ }^{3}$ Department of Public Health, Erasmus University Medical Center, Rotterdam, Netherlands

${ }^{4}$ Research Department of Epidemiology and Public Health, University College London, London, UK

${ }^{5}$ Department of Human Geography and Spatial Planning, Utrecht University, Utrecht, Netherlands

${ }^{6}$ Sorbonne Université, INSERM, Institut Pierre Louis d'Épidémiologie et de Santé Publique, Nemesis research team, Paris, France

${ }^{7}$ HUNT Research Centre, Department of Public Health and Nursing, Norwegian University of Science and Technology, Levanger, Norway

${ }^{8}$ Levanger Hospital, Nord-Trøndelag Hospital Trust, Levanger, Norway

${ }^{9}$ Department of Health Research Methods, Evidence, and Impact, McMaster

University, Hamilton, Canada

${ }^{10}$ McMaster Institute for Research on Aging, McMaster University, Hamilton, Canada

${ }^{11}$ Labarge Centre for Mobility in Aging, McMaster University, Hamilton, Canada

${ }^{12}$ Faculty of Nursing and Health Sciences, Nord Universitet-Levanger Campus,

Levanger, Norway

Twitter Tina Wey @tina_wey.

Acknowledgements The authors would like to thank Asli Gurer and Aliou Sarr for their contribution to data harmonisation work at Maelstrom Research; Dr Mauricio Avendano Pabon for general feedback on manuscript content; Dr Martijn Huisman for his assistance with data procurement; and Dr Ivet Bayes Marin, Professor Michael Dewey, Dr Lauren Griffith, Professor Scott Hofer, Dr Graciela Muniz Terrera and Dr Albert Sanchez Niubo for their invaluable input and expertise on the harmonisation of mental health and cognition data. This research was made possible using the data/

biospecimens collected by the Canadian Longitudinal Study on Aging (CLSA). The CLSA is led by Drs Parminder Raina, Christina Wolfson and Susan Kirkland. We also thank two anonymous reviewers for constructive feedback on an earlier version of the manuscript.

Contributors IF conceptualised the manuscript. TWW drafted and developed the manuscript with IF. DD, RW and GF contributed to manuscript development and content. $M A B, D D$ and RW coordinate the MINDMAP harmonisation infrastructure and database. $M A B, R W, I M, J M N$, MR and ET were the leads on harmonisation subject domains. Harmonisation leads and GF, AG and AS performed technical harmonisation work. IF coordinates the development of Maelstrom Research harmonisation guidelines and tools that were applied. FJvL and MA coordinate the MINDMAP project. FJvL, MB, BC, SK, PR, 
ERS and $\mathrm{MH}$ coordinate the included cohort studies and were involved in study design and data collection. MAB, IM, JMN, MR and ET were also members of cohort study teams. $M A B, I M, J M N, M R$, ET, FJvL, BC, PR and ERS provided critical feedback on the manuscript. All authors reviewed and approved the manuscript.

Funding MINDMAP is supported by the European Commission HORIZON 2020 Programme under grant agreement \#667661. The article does not reflect the Commission's views and in no way anticipates the Commission's future policy in this area. MAB was funded by a Netherlands Organization for Scientific Research (NWO) VENI grant on 'DenCityHealth: How to keep growing urban populations healthy?' (grant number 09150161810158). Funding for the Canadian Longitudinal Study on Aging (CLSA) is provided by the Government of Canada through the Canadian Institutes of Health Research (CIHR) under grant reference: LSA 94473 and the Canada Foundation for Innovation. The opinions expressed in this manuscript are the authors' own and do not reflect the views of the Canadian Longitudinal Study on Aging. PR holds the Raymond and Margaret Labarge Chair in Optimal Aging and Knowledge Application for Optimal Aging, is the Director of the McMaster Institute for Research on Aging and the Labarge Centre for Mobility in Aging, and holds a Tier 1 Canada Research Chair in Geroscience.

Competing interests None declared.

Patient consent for publication Not required.

Ethics approval Secondary use of cohort data for the MINDMAP harmonisation project received a declaration of no objection from the Medical Ethical Committee of Erasmus University Medical Centre. Data transfer agreements were established between all partners providing or analysing harmonised data. Cohort studies originally received informed consent of participants and ethical approval from their respective institutions. CLSA received ethical approval from the Hamilton Integrated Research Ethics Board. This research has been conducted using the CLSA data set, Baseline Comprehensive Dataset version 4.0, under Application Number 171013. GLOBE received a declaration of no objection from the Medical Ethical Committee of Erasmus MC. HAPIEE received ethical approval from the Joint UCL/UCLH Committees on the Ethics of Human Research. Ethical approval was also received from all local institutes: the Jagiellonian University's Committee on the Ethics of Clinical Research, the Kaunas Regional Biomedical Research Ethical Committee and the Institution Ethical Commission of the Czech Republic - National Institute of Public Health. HUNT received ethical approval from the Regional Committee for Medical Research Ethics, Mid-Norway, and MINDMAP-specific approval was received from the same committee. LASA received ethical approval by the Medical Ethical Committee of the Vrije Universiteit medisch centrum. RECORD received ethical approval from the Commission Nationale de I'Informatique et des Libertés.

Provenance and peer review Not commissioned; externally peer reviewed.

Data availability statement MINDMAP-harmonised data are not publicly available. Access to harmonised data for MINDMAP researchers is requested through the MINDMAP coordinators and from individual cohort studies following existing data-access policies. CLSA study-specific data are available from the Canadian Longitudinal Study on Aging (www.clsa-elcv.ca) for researchers who meet the criteria for access to de-identified CLSA data.

Supplemental material This content has been supplied by the author(s). It has not been vetted by BMJ Publishing Group Limited (BMJ) and may not have been peerreviewed. Any opinions or recommendations discussed are solely those of the author(s) and are not endorsed by BMJ. BMJ disclaims all liability and responsibility arising from any reliance placed on the content. Where the content includes any translated material, BMJ does not warrant the accuracy and reliability of the translations (including but not limited to local regulations, clinical guidelines, terminology, drug names and drug dosages), and is not responsible for any error and/ or omissions arising from translation and adaptation or otherwise.

Open access This is an open access article distributed in accordance with the Creative Commons Attribution 4.0 Unported (CC BY 4.0) license, which permits others to copy, redistribute, remix, transform and build upon this work for any purpose, provided the original work is properly cited, a link to the licence is given, and indication of whether changes were made. See: https://creativecommons.org/licenses/ by/4.0/

\section{ORCID iDs}

Tina W Wey http://orcid.org/0000-0002-1928-5881

J Mark Noordzij http://orcid.org/0000-0001-8580-1365

Milagros Ruiz http://orcid.org/0000-0001-7492-9873

Erik Timmermans http://orcid.org/0000-0002-3414-7196

Steinar Krokstad http://orcid.org/0000-0002-2932-6675

Erik Reidar Sund http://orcid.org/0000-0002-0736-5139

\section{REFERENCES}

1 Fortier I, Raina P, Van den Heuvel ER, et al. Maelstrom research guidelines for rigorous retrospective data harmonization. Int J Epidemiol 2017;46:103-5.

2 Beenackers MA, Doiron D, Fortier I, et al. MINDMAP: establishing an integrated database infrastructure for research in ageing, mental well-being, and the urban environment. BMC Public Health 2018;18:158

3 Raina PS, Wolfson C, Kirkland SA, et al. The Canadian Longitudinal Study on Aging (CLSA). Can J Aging 2009:28:221-9.

4 Kamphuis CBM, Turrell G, Giskes K, et al. Life course socioeconomic conditions, adulthood risk factors and cardiovascular mortality among men and women: a 17-year follow up of the GLOBE study. Int J Cardiol 2013;168:2207-13.

5 Peasey A, Bobak M, Kubinova R, et al. Determinants of cardiovascular disease and other non-communicable diseases in central and Eastern Europe: rationale and design of the HAPIEE study. BMC Public Health 2006;6:255.

6 Krokstad S, Langhammer A, Hveem K, et al. Cohort profile: the HUNT study, Norway. Int J Epidemiol 2013;42:968-77.

7 Huisman M, Poppelaars J, van der Horst M, et al. Cohort profile: the longitudinal aging study Amsterdam. Int J Epidemiol 2011;40:868-76.

8 Chaix B, Kestens Y, Bean K, et al. Cohort profile: residential and non-residential environments, individual activity spaces and cardiovascular risk factors and diseases: the RECORD cohort study. Int J Epidemio/ 2012;41:1283-92.

9 Doiron D, Marcon Y, Fortier I, et al. Software application profile: Opal and Mica: open-source software solutions for epidemiological data management, harmonization and dissemination. Int J Epidemiol 2017;46:1372-8.

10 Bergeron J, Doiron D, Marcon Y, et al. Fostering population-based cohort data discovery: the Maelstrom research cataloguing toolkit. PLOS One 2018;13: e0200926.

11 Fortier I, Burton PR, Robson PJ, et al. Quality, quantity and harmony: the DataSHaPER approach to integrating data across bioclinical studies. Int J Epidemiol 2010;39:1383-93.

12 RStudio Team. RStudio: integrated development environment for R. Boston, MA: RStudio, Inc, 2016. Available http://www.rstudio.com/

13 Core Team R. R: A language and environment for statistical computing. Vienna, Austria: R Foundation for Statistical Computing, 2019. Available https://www. R-project.org/

14 Timmermans EJ, Lakerveld J, Beulens JWJ, et al. Cohort profile: the Geoscience and Health Cohort Consortium (GECCO) in the Netherlands. BMJ Open 2018;8: e021597.

15 Rodgers SE, Demmler JC, Dsilva R, et al. Protecting health data privacy while using residence-based environment and demographic data. Health Place 2012:18:209-17.

16 MINDMAP harmonization project: Maelstrom research [Internet]. Available https:// www.maelstrom-research.org/mica/harmonization-study/mindmap-hp (accessed 21 Feb 2020)

17 MINDMAP R Markdown repository: GitHub [Internet]. Available https://github.com/ mindmap-cities ((accessed 20 Feb 2020)).

18 Fortier I, Doiron D, Burton P, et al. Invited commentary: consolidating data harmonization: how to obtain quality and applicability? Am J Epidemiol 2011;174:261-4. author reply 265-6.

19 Griffith LE, van den Heuvel E, Raina P, et al. Comparison of standardization methods for the harmonization of phenotype data: an application to cognitive measures. $A m$ J Epidemiol 2016;184:770-8.

20 Van den Heuvel ER, Griffith LE. Statistical harmonization methods in individual participants data meta-analysis are highly needed. Biom Biostat Int J 2016;3.

21 Friedenreich CM. Methods for pooled analyses of epidemiologic studies. Epidemiology 1993:4:295-302

22 Curran PJ, Hussong AM. Integrative data analysis: the simultaneous analysis of multiple data sets. Psychol Methods 2009;14:81-100.

23 Basagaña X, Pedersen M, Barrera-Gómez J, et al. Analysis of multicentre epidemiological studies: contrasting fixed or random effects modelling and meta-analysis. Int J Epidemiol 2018:47:1343-54.

24 Gelman A, Hill J. Data analysis using regression and multilevel/hierarchical models. New York, NY: Cambridge University Press, 2006.

25 Roux AVD, Mair C. Neighborhoods and health. Ann N Y Acad Sci 2010;1186:125-45.

26 InterConnect: global data for diabetes and obesity research: InterConnect [Internet]. Available http://www.interconnect-diabetes.eu/((accessed 22 Feb 2020)).

27 Doiron D, Burton P, Marcon Y, et al. Data harmonization and federated analysis of population-based studies: the BioSHaRE project. Emerg Themes Epidemio 2013;10:12

28 Sanchez-Niubo A, Egea-Cortés L, Olaya B, et al. Cohort profile: the Ageing Trajectories of Health: Longitudinal Opportunities and Synergies (ATHLOS) project. Int J Epidemiol 2019;48:1052-1053i.

29 Integrative Analysis of Longitudinal Studies of Aging (IALSA) [Internet]. Available http://www.ialsa.org/ (accessed 22 Feb 2020) 